\title{
Infrared laser multiphoton dissociation of proton-bound dimers of biomolecules: a new method to probe the acid-base properties of local sites in complex molecules
}

Sherrie Campbell, Jack L. Beauchamp

Sherrie Campbell, Jack L. Beauchamp, "Infrared laser multiphoton dissociation of proton-bound dimers of biomolecules: a new method to probe the acid-base properties of local sites in complex molecules," Proc. SPIE 1636, Applied Spectroscopy in Materials Science II, (14 May 1992); doi: $10.1117 / 12.59313$ 
Infrared laser multiphoton dissociation of proton bound dimers of biomolecules: a new method to probe the acid-base properties of local sites in complex molecules

Sherrie Campbell and J.L. Beauchamp

Contribution No. 8598 from the Arthur Amos Noyes Laboratory of Chemical Physics, California Institute of Technology, Pasadena, California 91125

\section{ABSTRACT}

New techniques such as matrix assisted laser desorption, electrospray ionization, and fast atom or ion bombardment make it possible to obtain quasimolecular (e.g. protonated) ions of thermally fragile biomolecules without significant decomposition or fragmentation. These ions can be studied using a variety of mass spectrometric techniques, most powerful among which is Fourier transform ion cyclotron resonance spectroscopy (FT-ICR). One of the important advantages of FT-ICR is the ability to store ions for long periods of time, facilitating studies of processes such as bimolecular reactions and laser photodissociation. One significant application of FT-ICR has been to study the acid-base properties of molecules in the gas phase. In this article we demonstrate a new method to probe the acid-base properties of local sites in biomolecules using a novel application of infrared multiphoton dissociation. The usual method of studying proton transfer reactions with appropriate reference bases does not work well with large molecules due to their propensity for radiative bimolecular cluster formation. The difficulty which this introduces can be circumvented by dissociating the proton bound dimer by infrared multiphoton excitation with a cw $\mathrm{CO}_{2}$ laser. Infrared heating assists dissociation along the lowest energy pathway and fragments the dimer to leave the proton preferentially on the more basic site. We illustrate this technique by demonstrating that the proton affinity of $\mathrm{N}$-acetyl glycine is intermediate between glycine and alanine.

\section{INTRODUCTION}

Identification of large involatile biological molecules by mass spectrometry has become a routine task as a result of improved experimental methodology for the formation of quasimolecular ions in which the species of interest is typically ionized by protonation, deprotonation or cationization (e.g. alkali ion attachment). Volatilization of a biomolecule from an appropriate matrix via laser desorption ${ }^{1}$, plasma desorption ${ }^{2}$, or fast atom or ion bombardment ${ }^{3}$ has become an efficient method for producing charged species. Electrospray and thermospray ionization ${ }^{4}$ are also useful methods for biomolecule analysis, often producing multiply charged quasimolecular ions. The addition of a proton or cation to a biological molecule may occur at a number of sites due to the many basic functional groups within the molecule. If the species is not too complex, it has proven useful in assessing protonation energetics of molecules to examine correlations of proton affinities with adiabatic lone pair ionization energies of basic sites. ${ }^{5}$ Such correlations can be used to predict the intrinsic base strengths of the sites and ascertain the site of protonation in simple molecules. It is difficult, however, to predict the site of protonation in complex molecules, such as proteins and peptides, since folding of the molecule can allow several basic sites to simultaneously interact with and solvate the charge center. In addition, the more usual methods for determining experimental proton or cation binding energies do not work well for large molecules as a result of the tendency to form adducts rather than undergo even exothermic proton or cation transfer reactions with reference bases. In this study we present a new technique which was developed to determine the acid-base properties of local sites in complex molecules.

Most methods which can be successfully employed to generate quasimolecular ions of biological molecules can be regarded as "soft" ionization, since the ions have low internal energies and little tendency to fragment. In fast atom bombardment (FAB), for example, some fragmentation occurs spontaneously upon ionization but the abundance of these ions is relatively low. While this is useful in the analysis of complex mixtures and the accurate determination of biomolecule molecular weights, it is often useful to have internal energies sufficiently high to promote fragmentation processes. For example, if sequencing of a peptide is desired, fragment ions are necessary to determine the amino acid composition. This cleavage is most often accomplished in mass spectrometers by collision induced dissociation (CID) where fragmentation results from energetic collisions with neutral atoms and molecules. Multiphoton excitation of molecules by continuous wave (cw) infrared radiation offers an alternative form of controlled molecular activation which has not been widely explored in applications to biomolecule mass spectrometry. 6,7 
The requirements to observe multiphoton dissociation with a $\mathrm{cw}$ infrared laser can be summarized as follows. The molecules must absorb in the region of accessible intense laser lines (9-11 microns for a $\mathrm{CO}_{2}$ laser). The density of states in the molecule must be sufficiently high to efficiently couple energy out of the pumped mode(s) and into other vibrational degrees of freedom where it becomes randomized, in competition with processes such as spontaneous and stimulated emission and collisional deactivation. This generally requires that the density of states exceeds $10^{3} / \mathrm{cm}^{-1}$, which is the inverse bandwidth of a flowing gas $\mathrm{CO}_{2}$ longitudinal discharge laser. This can generally be satisfied at room temperature with molecules having more than 10 heavy atoms and a thermal internal energy distribution. As shown in Fig. 1, the molecule continues to absorb energy through the pumped mode(s) until sufficient internal energy is accumulated to effect dissociation.

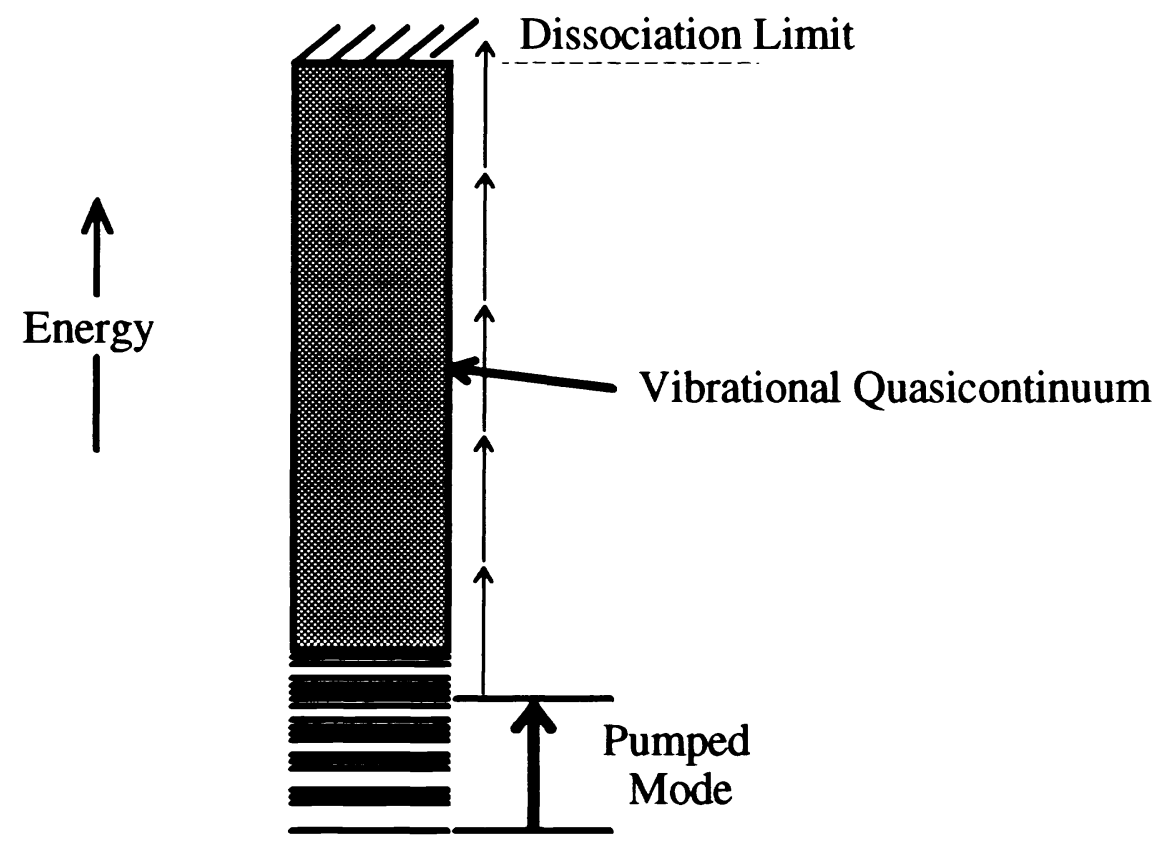

Fig. 1. Infrared multiphoton dissociation processes observed with cw lasers typically involve a pumped mode or modes which are strongly coupled to other vibrational modes in the molecule. Energy transfer out of the pumped mode is rapid and energy continues to accumulate until the dissociation limit is passed.

Infrared radiative cooling of large vibrationally excited molecules is significant on a 1 sec time scale. ${ }^{6}$ As a result, laser intensities must be sufficient to pump a significant fraction of the molecular population to levels of internal excitation where dissociation occurs on this time scale. The competing processes of excitation and deactivation are depicted in Fig. 2. Finally it is important to recognize that the energy in excess of the activation energy required to produce dissociation at a particular rate increases rapidly with increasing molecular size. These requirements can be quantified using RRKM calculations to describe the unimolecular reaction dynamics. ${ }^{8}$ It may be necessary to pump the internal energy distribution shown in Fig. 2 to many times the activation energy to achieve dissociation rates which can compete with radiative cooling or meet even more restrictive temporal constraints imposed by the mass spectrometric technique being used to examine the dissociation process. In time of flight and sector instruments, dissociation rates in excess of $10^{4}-10^{5} \mathrm{sec}^{-1}$ are required to effect fragmentation prior to detection. The time scale of trapped ion FT-ICR experiments is much longer, and dissociation rates of $1 \mathrm{sec}^{-1}$ can be accommodated.

The techniques of FT-ICR are uniquely suited for studying slow multiphoton dissociation of gas phase ions. ${ }^{9}$ In FT-ICR experiments ions may be trapped and irradiated under nearly collisionless conditions for times up to several seconds. The ability to detect and mass analyze reactants and products at any instant of time during the trapping and irradiation cycle 
provides direct information regarding reaction pathways and the kinetics of dissociation processes. Furthermore, both reactive and non-reactive (buffer) gases can be added to allow for the effects of collisions during the storage period to be observed. We believe the $\mathrm{cw}$ infrared laser multiphoton activation of gas-phase biological ions holds great promise as a means of controlled molecular activation to be used in conjunction with experiments designed to probe the properties and structures of complex biological molecules.

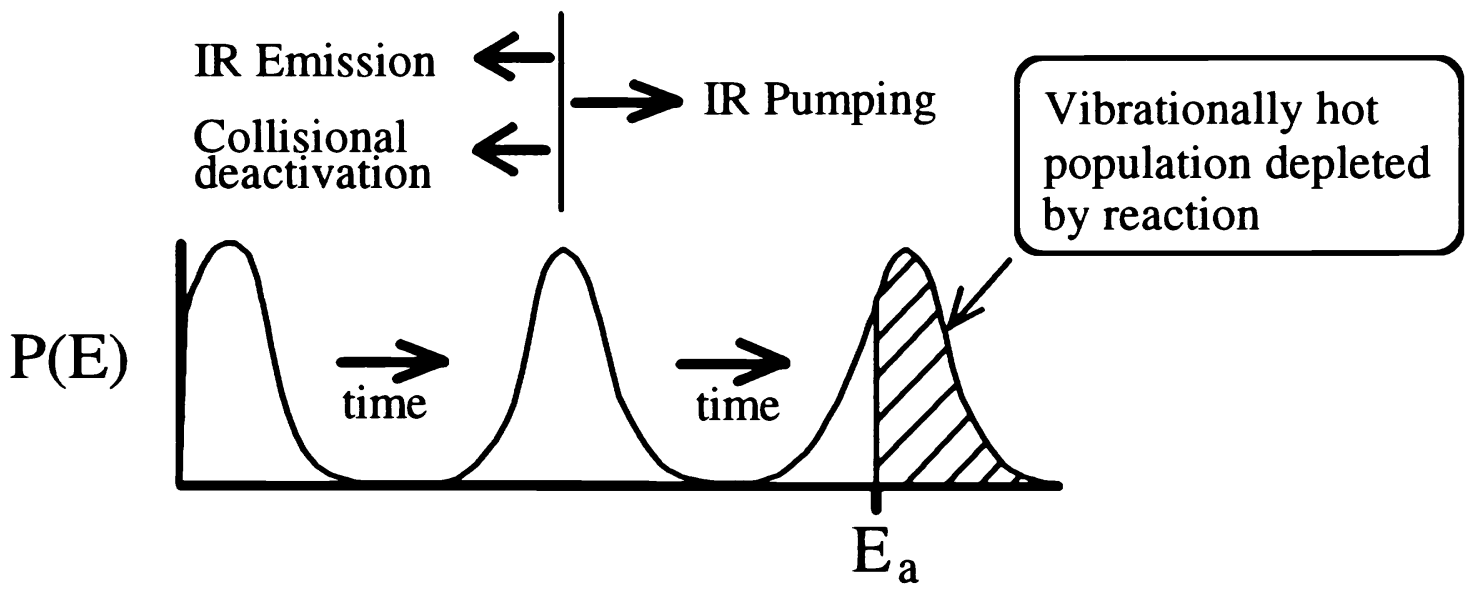

\section{Vibrational Energy $(\mathrm{E}) \quad \longrightarrow$}

Fig. 2. Schematic illustration of molecular vibrational energy distribution during infrared laser multiphoton dissociation processes. IR pumping competes with IR emission and collisional deactivation. With large molecules the energy distribution may have to significantly exceed the activation energy before dissociation can compete with IR emission.

In this paper, we demonstrate the application of $\mathrm{cw}$ infrared laser multiphoton dissociation (IRMPD) of model biological systems to determine the acid-base properties of local sites in complex molecules, by examining the preferred direction of dissociation of proton bound dimers of the molecule of interest with appropriate reference bases. The gas phase proton affinity of a base, $B_{1}$, is defined as the negative of the enthalpy change for reaction (1).

$$
\mathrm{B}_{1}+\mathrm{H}^{+} \rightarrow \mathrm{B}_{1} \mathrm{H}^{+}
$$

The usual method employed to determine proton affinities of molecules in the gas phase involves observation of proton transfer equilibria such as shown in reaction (2), where $B_{2}$ is a reference species with known proton affinity. ${ }^{10}$

$$
\mathrm{B}_{1} \mathrm{H}^{+}+\mathrm{B}_{2} \rightarrow \mathrm{B}_{2} \mathrm{H}^{+}+\mathrm{B}_{1}
$$

With sufficiently large molecules the clustering process (3) can become important, and can even occur as a bimolecular process if the initially formed proton bound dimer is radiatively stabilized by infrared emission before it can dissociate. ${ }^{11}$

$$
\mathrm{B}_{1} \mathrm{H}^{+}+\mathrm{B}_{2} \rightarrow \mathrm{B}_{1} \mathrm{~B}_{2} \mathrm{H}^{+}
$$

The present study starts with stable proton bound dimers and examines the kinetics of the competitive dissociation processes (4) and (5), using infrared laser multiphoton excitation to activate the proton bound dimer.

$$
\begin{aligned}
\mathrm{B}_{1} \mathrm{~B}_{2} \mathrm{H}^{+}+\mathrm{nh} \nu & \rightarrow \mathrm{B}_{1} \mathrm{H}^{+}+\mathrm{B}_{2} \\
& \rightarrow \mathrm{B}_{2} \mathrm{H}^{+}+\mathrm{B}_{1}
\end{aligned}
$$


Our results exemplify the temporal constraints associated with this experimental methodology and serve as an example for future quantitative studies involving IRMPD.

\section{EXPERIMENTAL}

Experimental techniques associated with ion cyclotron resonance spectroscopy (ICR) and in particular FT-ICR, have been previously described in detail. ${ }^{9}$ Experiments were performed using an IonSpec FT-ICR data system in conjunction with a 1-inch cubic trapping cell modified for infrared photochemistry. ${ }^{12}$ Experiments were carried out at 2 Tesla using a Varian 15-inch electromagnet.

Appreciable vapor pressure of the amino acids was generated by heating a quartz tube of solid sample in vacuum directly under the trapping cell. Because of this arrangement, neutral pressures could not be accurately determined. Based on the observed reaction kinetics and assuming typical ion-molecule reaction rates, we estimate that sample pressures were in the range $10^{-7}-10^{-8}$ Torr. A typical experimental pulse sequence is shown in Fig. 3. Positive ions were generated by electron impact ionization, using a nominal electron beam energy of 15 to 20 volts. Ions were stored in the trapping cell and massselected by a series of ion ejection pulses. The unfocused infrared beam from a grating tuned Apollo $550 \mathrm{~A} \mathrm{cw} \mathrm{CO}_{2}$ laser enters the vacuum system through a $\mathrm{NaCl}$ window $(25.4 \mathrm{~mm}$ diameter $\times 3.5 \mathrm{~mm}$ thick) and passes through a $92 \%$ transmittance mesh to irradiate trapped ions. The transmitter plate reflects the beam for a second pass through the iontrapping region and out of the apparatus. The $\mathrm{CO}_{2}$ laser beam is externally triggered by a control pulse from the IonSpec data system. Several infrared multiphoton dissociation spectra of mass-selected proton bound dimer ions considered in this study were obtained at a constant laser intensity, but showed no dependence on wavelength. Dissociation rates were measured for a range of laser powers, determined using a Scientech surface absorbing disc calorimeter.

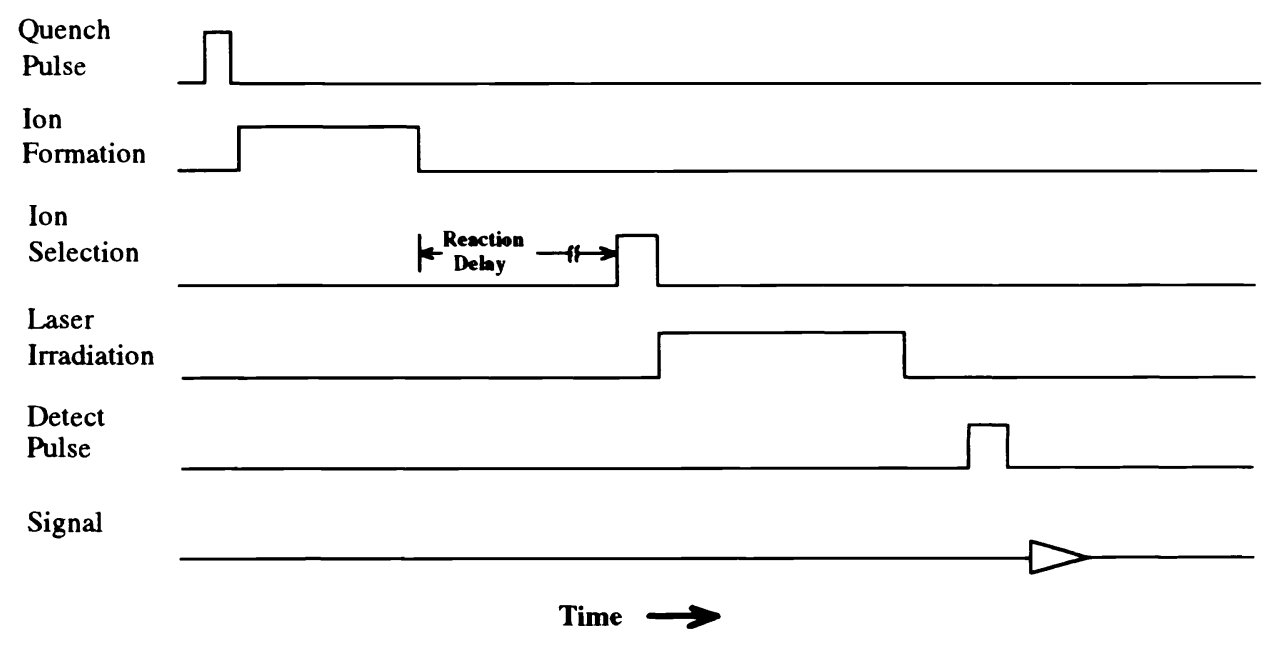

Fig. 3. Trapped ion timing sequence for studies of infrared laser multiphoton dissociation processes. The quench pulse removes residual ions from the FT-ICR cell. Ion formation involves pulsing the electron beam to form ions from the gaseous sample. After a suitable reaction delay, the desired ion is isolated and subjected to laser irradiation.

\section{RESULTS AND DISCUSSION}

\subsection{Reactions of $\mathrm{N}$-acetyl glycine with alanine}

Meot-Ner measured the gas phase proton affinity of $\mathrm{N}$-acetyl glycine methyl ester and found it slightly larger than the proton affinity of alanine ${ }^{13}$. It was predicted from those results that the acid, $\mathrm{N}$-acetyl glycine, would have a proton affinity similar to or slightly smaller than that of alanine. Since proton affinities can be extracted from proton transfer reaction data, experiments were initially attempted which would establish gas phase proton transfer equilibria in mixtures of $\mathrm{N}$-acetyl glycine with alanine $(\mathrm{PA}=214.8 \mathrm{kcal} / \mathrm{mol})$ and glycine $(\mathrm{PA}=212.0 \mathrm{kcal} / \mathrm{mol}) .^{5}$ 
<smiles>CC(N)C(=O)O</smiles>

Alanine<smiles>CC(=O)NCC(=O)O</smiles>

$\mathrm{N}$-acetyl Glycine<smiles>NCC(=O)O</smiles>

Glycine

Proton exchange equilibrium between $\mathrm{N}$-acetyl glycine and alanine could not be established due to radiative bimolecular cluster formation. Protonated molecules clustered with the neutral background gas in the vacuum chamber and created primarily heterodimers of $\mathrm{N}$-acetyl glycine and alanine (Fig. 4). It is of particular interest that only the heterodimer is observed in abundance, suggesting that it is inherently more stable than the two homodimers which can be formed in this mixture. This suggests that some type of synergistic hydrogen bonding gives this species special stability. The proton bound heterodimer was isolated by ejecting all remaining lower and higher mass ions from the FT-ICR cell and dissociated by infrared multiphoton excitation with a cw $\mathrm{CO}_{2}$ laser (Fig. 5). The dissociation products detected were the intact protonated alanine and $\mathrm{N}$-acetyl glycine ions. Only the weaker hydrogen bonds which hold the complex together are broken. More complex processes involving extensive rearrangement were not observed. The relative abundances of the two product ions were not equivalent but were in a ratio of approximately 4:1 alanine: $\mathrm{N}$-acetyl glycine. To the extent that it could be accurately determined this ratio did not vary significantly with either laser wavelength or power. Originally it was naively hoped that at low laser intensities only a single product would be observed. The quantitative analysis of the dissociation kinetics presented below makes it clear why this does not happen and might not be expected even if the proton affinity differences were large compared to the energy of a single infrared photon $(2.7 \mathrm{kcal} / \mathrm{mol})$.

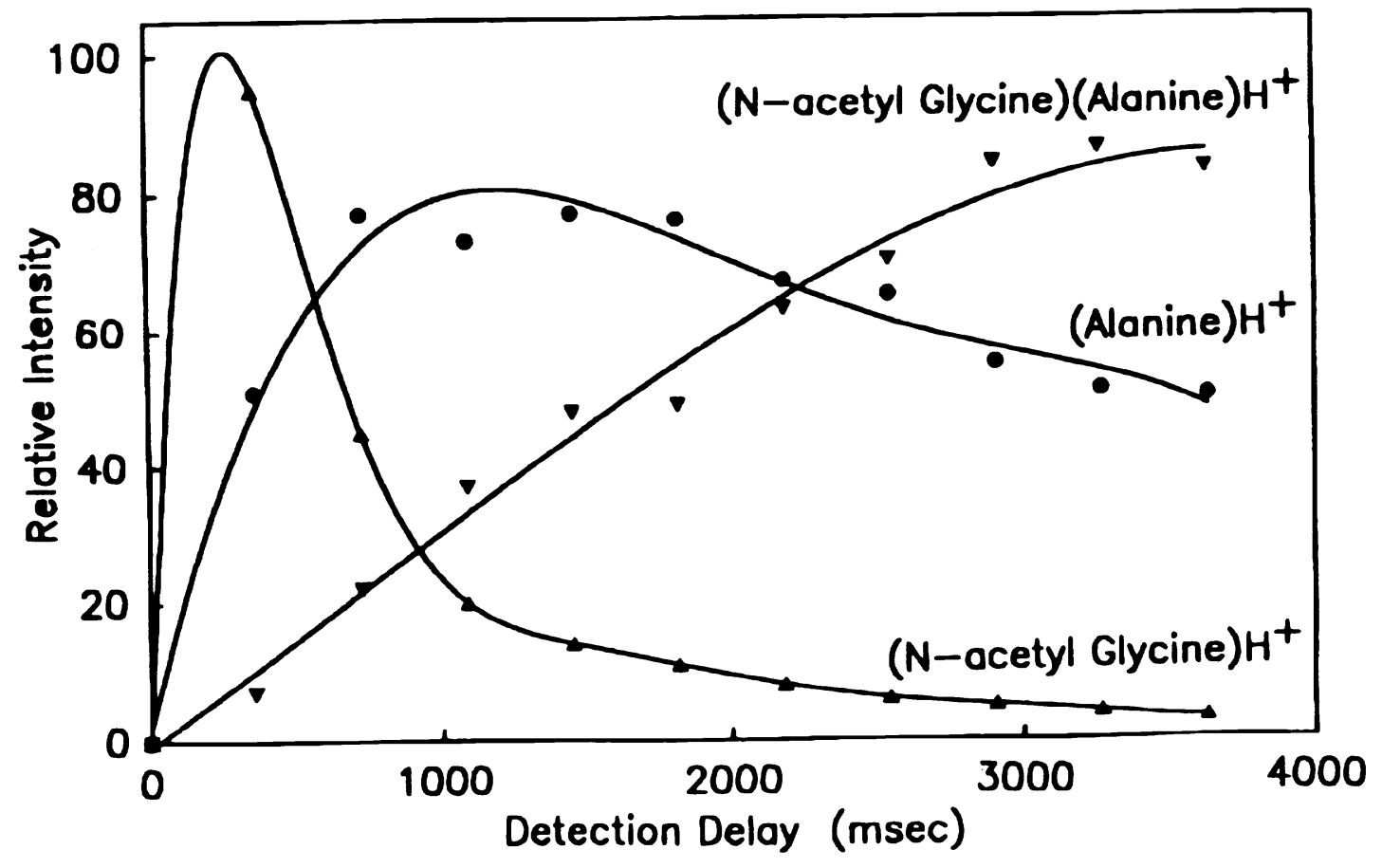

Fig. 4. Variation of ion abundance with time following ionization of a mixture of $\mathrm{N}$ acetyl glycine and alanine by electron impact $(15 \mathrm{eV})$. A variety of different ions (not shown) are formed which react to give the two protonated molecules. The heterodimer is the major ion observed at long times. 


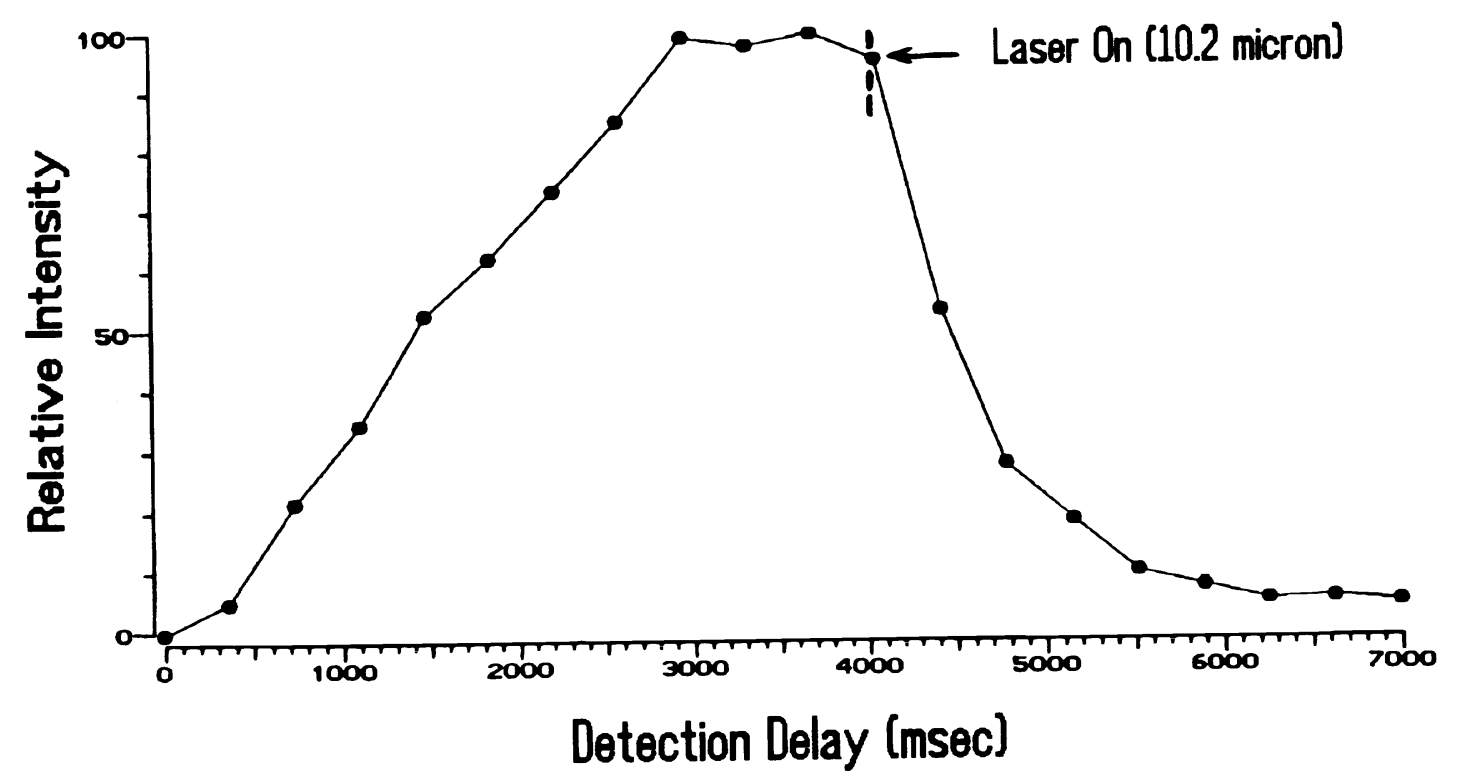

Fig. 5. Intensity of proton bound dimer of $\mathrm{N}$-acetyl glycine and alanine as a function of time. The laser $\left(15 \mathrm{~W} \mathrm{~cm}^{-2}\right)$ is switched on at $4 \mathrm{sec}$ and the dimer intensity decreases with time as a result of multiphoton dissociation.

\subsection{Reactions of $\mathrm{N}$-acetyl glycine with glycine}

The gas phase proton affinity of $\mathrm{N}$-acetyl glycine was predicted to be slightly higher than the proton affinity of glycine, due to the ability of the acetylated molecule to solvate the charge with an intramolecular hydrogen bond. Proton transfer equilibrium studies to determine the more basic of the two species produced results similar to those described above, with formation of a proton bound heterodimer as the primary product. Infrared multiphoton dissociation of the cluster produced protonated ions of intact $\mathrm{N}$-acetyl glycine and glycine molecules with no additional fragmentation. Product ion abundances of 2.5:1 N-acetyl glycine:glycine were observed and both protonated species were detected at the lowest laser intensities. These experiments suggest that the proton affinity of $\mathrm{N}$-acetyl glycine is intermediate between glycine and alanine.

\subsection{Photodissociation kinetics and the temporal constraints for $\mathrm{cw}$ laser infrared multiphoton dissociation}

As shown in Fig. 5, the proton bound dimer of $\mathrm{N}$-acetyl glycine and alanine readily undergoes IRMPD with modest laser intensities. This is not surprising since a molecule of this complexity should easily be in the vibrational quasicontinuum at room temperature. Evaluation of the photodissociation kinetics is straightforward using data such as shown in Fig. 5 for the disappearance of the proton bound dimer after the laser is switched on. Results are shown in Fig. 6 for several laser intensities, and the rates taken from the slopes are shown plotted in Fig. 7 as a function of laser irradiance (expressed in W $\mathrm{cm}^{-2}$ ). The data in Fig. 7 show that the dissociation rate approaches zero with irradiances below $5 \mathrm{~W} \mathrm{~cm}^{-2}$. Note that this corresponds to photodissociation rates which drop below $1 \mathrm{sec}^{-1}$. As noted in the introduction, unless the dissociation rates exceed this value, infrared emission will lead to significant relaxation of the vibrationally excited molecules in competition with laser pumping. 


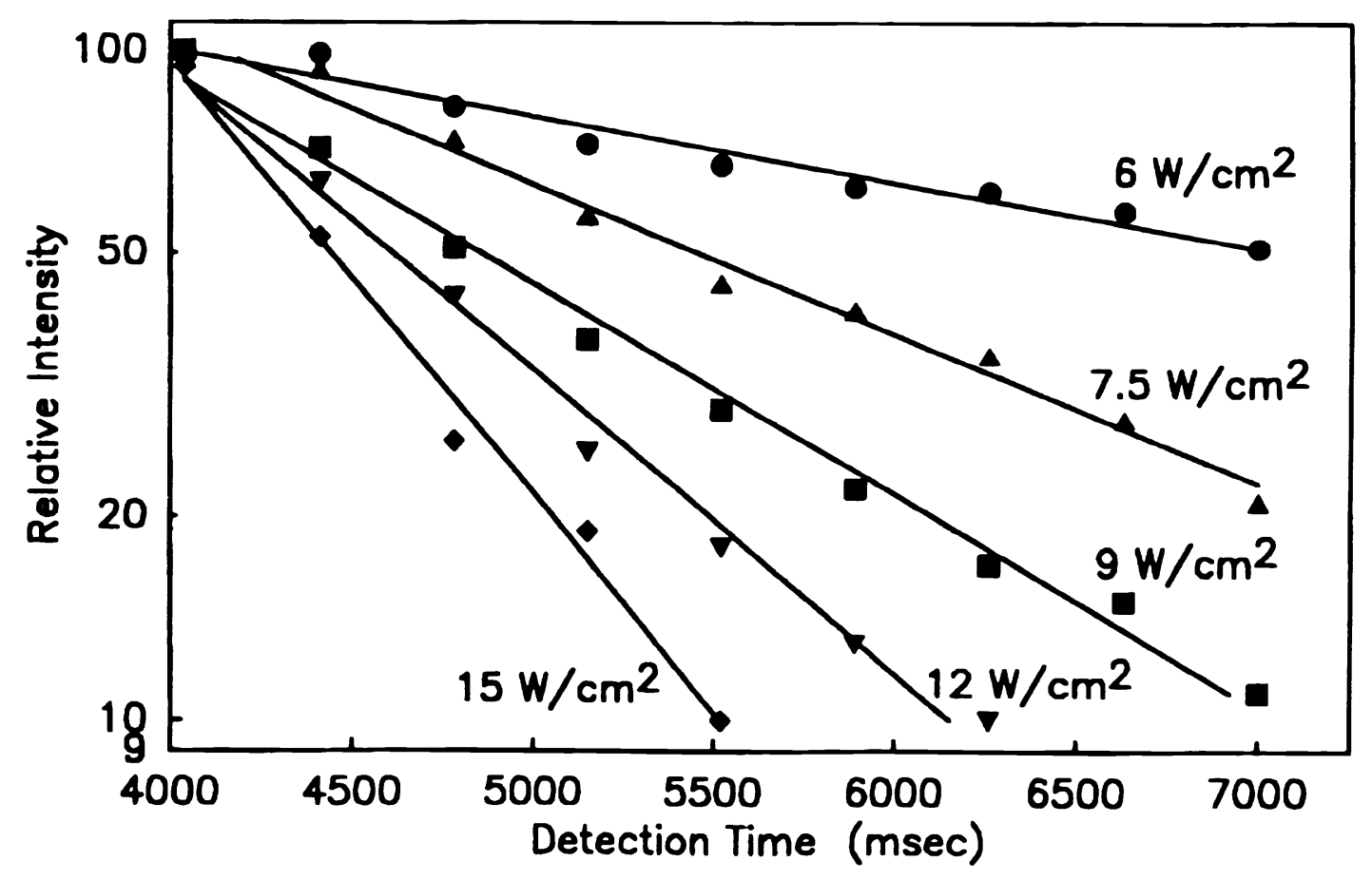

Fig. 6. Semilog plot showing the decay of the proton bound dimer of $\mathrm{N}$-acetyl glycine and alanine after the infrared laser is turned on. The resulting rate (taken from the slope) increases with increasing laser intensity. Other conditions are as shown in Fig. 5.

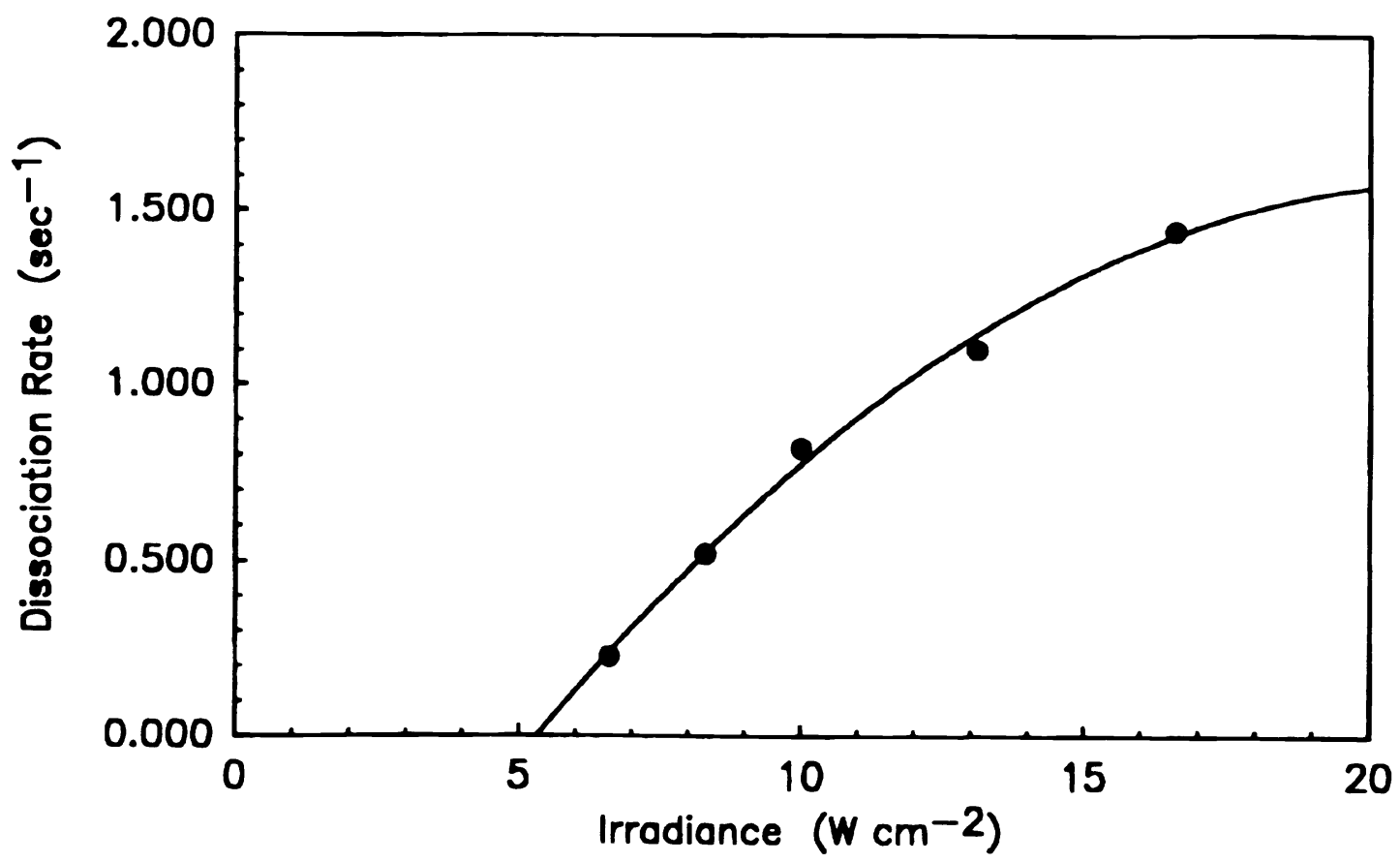

Fig. 7. Variation of infrared laser multiphoton dissociation rate of the proton bound dimer of $\mathrm{N}$-acetyl glycine and alanine with laser irradiance. The rate approaches zero with irradiance levels below $5 \mathrm{~W} \mathrm{~cm}^{-2}$ due to cooling of the proton bound dimer by infrared emission. 
The dissociation kinetics can be further quantified using RRKM calculations to model this process. The hydrogen bond strength between a protonated oxygen (the carbonyl of the amide linkage in $\mathrm{N}$-acetyl glycine) and a basic site on alanine (either the nitrogen or the carbonyl oxygen) is approximately $30 \mathrm{kcal} / \mathrm{mol}$, which is taken to be the activation energy for the process leaving the proton on the more basic site (alanine). We take $31.7 \mathrm{kcal} / \mathrm{mol}$ as the activation energy for the higher energy process in which the proton is retained on $\mathrm{N}$-acetyl glycine. Using the Whitten-Rabinovitch approximation to evaluate state densities for both the energized molecule and the activated complex gives the predicted rates as a function of excess energy shown in Fig. 8.

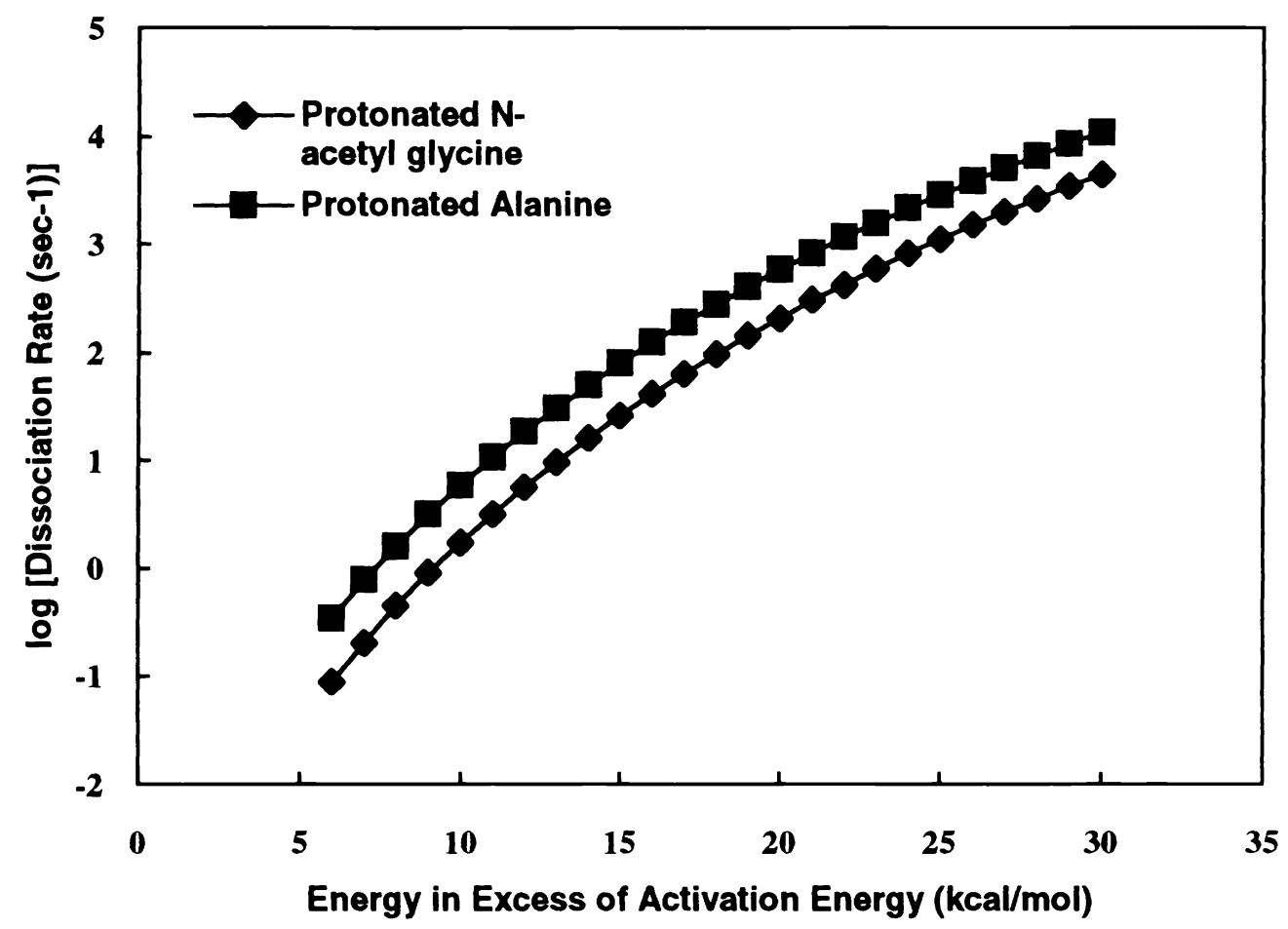

Fig. 8. RRKM calculations for the rate of dissociation of the proton bound dimer of $\mathrm{N}$ acetyl glycine and alanine. Activation energies for the two processes of 31.7 and 30.0 $\mathrm{kcal} / \mathrm{mol}$ are assumed, respectively.

The model calculations shown in Fig. 8 illustrate several points. First, in agreement with our experimental observations, the ratio of the two products varies only minimally with excess energy, from 4.0 at $10 \mathrm{kcal} / \mathrm{mol}$ to $3.5 \mathrm{at} 15 \mathrm{kcal} / \mathrm{mol}$, which encompasses the range of dissociation rates considered in our experiments (Fig. 7). To produce dissociation at a rate which can compete with radiative cooling, the energy in excess of the activation energy must be approximately 10 $\mathrm{kcal} / \mathrm{mol}$, which is large in comparison to the difference in activation energy assumed. With $2.7 \mathrm{kcal} / \mathrm{mol}$ for each photon, this corresponds to absorption of roughly 4 photons in excess of the threshold energy requirement. The difference in activation energies assumed for this calculation was chosen to reproduce the observed product ratio. Similar RRKM calculations were carried out for the other heterodimer observed ( $\mathrm{N}$-acetyl glycine and glycine) and a proton affinity difference of $1.1 \mathrm{kcal} / \mathrm{mol}$ can reproduce the observed product distribution reasonably well. We believe that the more quantitative analysis permitted by the RRKM calculations makes it possible to extract approximate values for the proton affinity that are more accurate than would be yielded by simple bracketing experiments. In some respects these experiments are similar to quantitative analysis of competitive fragmentation processes observed in metastable ion studies. $^{14}$ 


\section{CONCLUSIONS}

In this study we have demonstrated a novel application of IRMPD to determine the acid base properties of biological molecules. The proton affinity of $\mathrm{N}$-acetyl glycine has been determined to be intermediate between glycine and alanine, with a value of $213.1 \pm 0.5 \mathrm{kcal} / \mathrm{mol}$ being more closely determined by RRKM model calculations. By forming solvated adducts of biological molecules with appropriate reference bases it should be possible to extend these studies to more complex systems. We have recently completed construction of an external ion source FT-ICR spectrometer with a 7 Tesla superconducting magnet. With this system it is possible to separate the regions in which ion formation and ion trapping occur. This facilitates studies of IRMPD under truly collision free conditions and many such studies are in progress.

This study serves to illustrate the temporal constraints associated with IRMPD using $\mathrm{cw}$ infrared lasers. The requirement that dissociation rates exceed $1 \mathrm{sec}^{-1}$ does not sound terribly demanding until one considers what it means for a large biological molecule. We have carried out model calculations which indicate that species in the molecular weight range around 10,000 Daltons typically require over $100 \mathrm{eV}$ internal energy to yield reasonable dissociation rates! Achieving the controlled fragmentation of such molecules presents a formidable challenge.

\section{ACKNOWLEDGMENTS}

The authors wish to thank K. Irikura for his assistance in designing the sample volatilization source. We are indebted to Dr. Arnold Beckman and the Beckman Institute at Caltech for their support of our studies of biomolecule mass spectrometry. We gratefully acknowledge the financial support of Sherrie Campbell from a NIH-NRSA traineeship in Biotechnology. This work was supported in part by the National Science Foundation under grant CHE-9108318.

\section{REFERENCES}

${ }^{1}$ M. Karas and F. Hillenkamp, "Laser Desorption Ionization of Proteins with Molecular Masses Exceeding 10,000 Daltons," Anal. Chem. vol. 60, pp. 2299-2301, 1988.

${ }^{2}$ R. D. Macfarlane, "Role of Intermolecular Interactions in the Desorption of Molecular Ions from Surfaces," Desorption Mass Spectrometry: Are SIMS and FAB the Same? Editor P.A. Lyon, ACS Symposium Series vol. 291, pp. 56-68, American Chemical Society, Washington, D.C. 1985.

${ }^{3}$ M. Barber, R.S. Bordoli, R.D. Sedgewick and A.N. Tyler, "Fast Atom Bombardment of Solids: A New Ion Source for Mass Spectrometry," J. Chem. Soc. Chem. Comm. pp. 325-328, 1981.

${ }^{4} \mathrm{~J}$. B. Fenn, M. Mann, C.K. Meng, S.F. Wong and C. M. Whitehouse, "Electrospray Ionization for Mass Spectrometry of Large Biomolecules," Science, vol. 246, pp. 64-71, 1989.

${ }^{5}$ S. Campbell and J.L. Beauchamp, "Correlations of Lone Pair Ionization Energies with Proton Affinities of Amino Acids and Related Compounds. Site Specificity of Protonation," Int. J. Mass Spectrom. and Ion Processes, in press, 1992.

${ }^{6}$ L. R. Thorne and J.L. Beauchamp, "Infrared Photochemistry of Gas Phase Ions," Gas Phase Ion Chemistry, Editor M. T. Bowers, vol. 3, pp. 41-97, Academic Press, New York, 1984.

${ }^{7}$ C. H. Watson, G. Baykut, and J. R. Eyler, "Infrared Multiphoton Dissociation of Laser-Desorbed Ions," Fourier Transform Mass Spectrometry, Edited by M. V. Buchanan, ACS Symposium Series vol. 359, pp. 140-154, American Chemical Society, Washington, D.C., 1987.

${ }^{8}$ P.J. Robinson and K.A. Holbrook, Unimolecular Reactions, Wiley-Interscience, Bristol, England, 1972.

${ }^{9}$ A. G. Marshall, "Fourier Transform Ion Cyclotron Resonance Mass Spectrometry,". Acc. Chem. Res., vol. 18, pp. 316323, 1984.

${ }^{10}$ J. F. Wolf, R. H. Staley, I. Koppel, M. Taagepera, R. T. McIver, Jr., J. L. Beauchamp, and R. W. Taft, "Gas Phase Basicities and Relative Proton Affinities of Compounds between Water and Ammonia from Pulsed Cyclotron Resonance Thermal Equilibria Measurements," J. Am. Chem. Soc., vol. 99, pp. 5417-5429, 1977.

${ }^{11}$ R. L. Woodin and J.L. Beauchamp, "Bimolecular Infrared Radiative Association Reactions. Attachment of $\mathrm{Li}^{+}$to Carbonyl Compounds in the Gas Phase," Chem. Phys. vol. 41, pp.1-9, 1979.

${ }^{12} \mathrm{~S}$. K. Shin and J. L. Beauchamp, "Identification of $\mathrm{Mn}(\mathrm{CO}) \mathrm{n}_{\mathrm{CF}_{3}^{-}}(\mathrm{n}=4,5)$ Structural Isomers by IR Multiphoton Dissociation, Collision-Induced Dissociation, and Specific Ligand Displacement Reactions: Studies of the Trifluoromethyl Migratory Decarbonylation Reaction in the Gas Phase," J. Am. Chem. Soc., vol. 112, pp 2057-2066, 1990. 
${ }^{13}$ M. Meot-Ner, "The Ionic Hydrogen Bond. 4. Intramolecular and Multiple Bonds. Protonation and Complexes of Amides and Amino Acid Derivatives," J. Am. Chem. Soc., vol. 106, pp 278-283, 1984.

${ }^{14} \mathrm{G}$. Bojesen , "The Order of Proton Affinities of the 20 Common L- $\alpha$-Amino Acids," J. Am. Chem. Soc., vol. 109, pp. 5578-5558, 1987.

210 / SPIE Vol. 1636 Applied Spectroscopy in Materials Science II (1992) 